\title{
Meta
}

Journal des traducteurs

Translators' Journal

\section{PÉCHOIN, Daniel (dir.) (1991): Thésaurus Larousse. Des mots \\ aux idées, des idées aux mots, Paris, Larousse, XXI + 146 p.}

\section{Jean-Claude Boulanger}

Volume 38, numéro 1, mars 1993

La traduction et l'interprétation dans le nord du Canada

Translation and Interpretation in Northen Canada

URI : https://id.erudit.org/iderudit/002120ar

DOI : https://doi.org/10.7202/002120ar

Aller au sommaire du numéro

Éditeur(s)

Les Presses de l'Université de Montréal

ISSN

0026-0452 (imprimé)

1492-1421 (numérique)

Découvrir la revue

\section{Citer cet article}

Boulanger, J.-C. (1993). PÉCHOIN, Daniel (dir.) (1991) : Thésaurus Larousse. Des mots aux idées, des idées aux mots, Paris, Larousse, XXI + 146 p. Meta, 38(1),

130-136. https://doi.org/10.7202/002120ar d'utilisation que vous pouvez consulter en ligne.

https://apropos.erudit.org/fr/usagers/politique-dutilisation/ 
PÉCHOIN, Daniel (dir.) (1991): Thésaurus Larousse. Des mots aux idées, des idées aux mots, Paris, Larousse, XXI + $1146 \mathrm{p}$.

Encore un dictionnaire qui tente de percer un marché déjà fort encombré, sinon saturé, dira-t-on à la vue de ce nouveau produit Larousse. Doublement nouveau d'ailleurs: d'une part, parce que l'ouvrage est récent, d'autre part, parce qu'il s'agit d'un genre tout à fait inédit en français contemporain, donc un pari éditorial et un investissement financier d'importance. Encore un thésaurus diront les langagiers de LSP. Pour quoi faire? ceux des banques de terminologie ne sont-ils pas déjà riches et accessibles à distance? Qu'a donc de différent, de si particulier, celui qu'on nomme le Thésaurus Larousse? Et, qu'est-ce donc qu'un thésaurus? Où doit-on le situer dans la grande hiérarchie dictionnairique?

Nous sommes tous et toutes habitués à voyager activement ou paresseusement dans des dictionnaires de mots et dans des dictionnaires de choses - les encyclopédies - ou dans un amalgame des deux catégories, dont l'aboutissement le plus accompli est sans aucun doute le Petit Larousse illustré. En revanche, en tant que francophones, nous ne fréquentons guère les répertoires généraux complets associant les mots et les idées, les 
idées et les mots. Malgré quelques précédents historiques et quelques productions de dictionnaires analogiques (par exemple, Boissière, en 1862; Maquet, en 1936), le thésaurus ne fait pas partie des outils consultés automatiquement dès que survient une interrogation sur la langue. Seuls les terminologues et les documentalistes sont des familiers de cette sorte de recueil, mais pour d'autres raisons. On n'a donc pas bâti de tradition en langue française, comme cela s'est fait dans d'autres communautés linguistiques, notamment chez les anglophones pour qui l'œuvre de Roget n'a plus de secret.

Les éditions Larousse suppléent à cette grande (grave?) lacune - mais peut-on vraiment parler de manque ou d'absence lorsque le besoin ne s'est pas manifesté auparavant? - en publiant un thésaurus de la langue. Ce thésaurus-dictionnaire se fixe comme objectif de permettre à des usagers aux profils diversifiés (journalistes, publicitaires, rédacteurs, orateurs, enseignants, étudiants, collégiens, etc.) de métamorphoser leurs idées en mots et de filer leurs mots sur de nouvelles idées. Ces allers-retours s'accompliront en comparant des champs de signification qui englobent aussi bien les synonymes, que les antonymes et les analogies, en somme, tout le réseau de structuration onomasiologique, principe auquel tous les terminologues font appel dans leurs travaux. On comprendra donc qu'un thésaurus est d'abord et avant tout un instrument d'aide à la pensée construit sur des fondements sémantiques, contrairement au dictionnaire de langue, dans lequel une forme linguistique se voit greffer un ou des sens qui lui conviennent.

Le Thésaurus Larousse (TL) est d'une présentation matérielle tout à fait originale et exceptionnelle: le livre est solide, bien conçu, le format est aisément maniable et son design, très agréable, accroche l'œil. Les informations contenues dans les articles sont disposées en colonnes, deux pour le dictionnaire, quatre pour l'index; la typographie est soignée, claire et aérée et elle facilite la lisibilité - au sens pédagogique — de l'ouvrage. Outre les discours introductifs habituels (préface, mode d'emploi, etc.) et les diverses listes d'abréviations, la matière du TL est divisée en trois grandes parties : le sommaire, le dictionnaire, l'index, dont l'interface stratégique est permanente. Voici une brève analyse de chacune.

\section{LE SOMMAIRE (PP. XV-XXI)}

Le sommaire fournit une vue synthétique du classement privilégié dans les «articles». Dans ce chapitre, le plan d'organisation fait voir trois grandes divisions qui servent à rendre la structure de l'Univers, à savoir A. LE MONDE, B. L'HOMME, C. LA SOCIÉTÉ. Ce schéma de présentation logique est classique dans les recherches de type onomasiologique à caractère exhaustif. Il permet d'embrasser l'ensemble des connaissances humaines. L'architecture du TL prolonge celles que l'on retrouve dans des ouvrages avec lesquels les philologues sont accoutumés de travailler, comme celui de Rudolf Hallig et Walther von Wartburg: Système raisonné des concepts pour servir de base à la lexicographie, publié en 1963.

Chaque grand ensemble du dictionnaire est lui-même l'objet de sous-ensembles qui, à leur tour, et suivant les besoins, se subdivisent en sous-paragraphes. Ainsi que fait observer D. Péchoin dans sa préface, les «ramifications terminales de cette classification arborescente sont constituées par les 873 articles qui forment la substance vive de l'ouvrage» (p. v). Si l'on consulte la partie B. L'HOMME, on verra qu'elle comprend quatre grandes divisions: I. GÉNÉRALITÉS, II. LE CORPS, III. L'ESPRIT, IV. LA VOLONTÉ ET L'ACTION. La matière de la section II. LE CORPS est distribuée sous six intertitres: 1. Parties du corps, 2. Fonctions corporelles, 3. Perception, 4. États du corps, 5. Santé et maladie, 6. Médecine. La sous-section 3. Perception raffine davantage la structuration avec ses six blocs: a. Généralités, b. Vision, c. Ouïe, d. Odorat, e. Goût, f. Toucher. Enfin, dans chacun des blocs se trouve la liste des indispensables 
mots clés qui constituent en quelque sorte les descripteurs ou les super-concepts sous lesquels sont rangées toutes les autres informations (ex.: sous b. Vision, il y a 17 mots clés ou paragraphes numérotés : 346 Vision, 347 Troubles de la vision, 348 Visibilité, 349 Invisibilité, [...], 362 Polychromie).

En résumé, le schéma structurel du TL se définit comme suit: C.I.1.a.581, qui se décode ainsi :

\author{
C. LA SOCIÉTÉ \\ I. LES RELATIONS SOCIALES \\ 1. Rapports entre personnes \\ a. Relations \\ 581 Sociabilité \\ $[\ldots]$ \\ 599 Dureté
}

Curieusement, ce sommaire n'est pas repris dans le corps du thésaurus. On n'y retrouve en effet que les 873 dénominations de tête, alignées les unes à la suite des autres, sans que soient intercalés les titres ou les intertitres. Le lecteur passe du numéro 580 Violence à 581 Sociabilité, sans que soient indiqués ni le changement de grande division (de B. à C.) ni ceux de section, de sous-section, etc. (de B.IV.2.f.580 à C.I.1.a.581). La raison tient peut-être au fait que le TL fut rédigé directement à l'écran et que le programme informatique ne permettait pas de fragmenter la suite des articles afin d'y insérer les titres et les intertitres. Il semble cependant qu'il faille voir là un choix méthodologique délibéré, qui se justifie par le fait de ne pas vouloir interrompre la séquence des paragraphes numérotés.

\title{
2. LE DICTIONNAIRE (PP. 1-629)
}

Le corps proprement dit du dictionnaire est formé d'une nomenclature de 873 superentrées qui sont ordonnées par grandes familles conceptuelles auxquelles un mot-idée donne accès (ex. : existence, indépendance, rotation, astronomie). Les 873 mots-vedettes sont tous représentés par des substantifs simples ainsi que par quelques unités reliées par et (ex.: 290 Herbes et fougères, 292 Mousses et hépatiques) ou par quelques rares unités complexes (ex.: 347 Troubles de la vision, 370 Son grave, 671 Systèmes politiques, 783 Instruments de musique) sous lesquels sont fédérés autant d'articles - appelés paragraphes par les auteurs. Ce volet de 630 pages recense l'ensemble des mots relevant des différentes sphères notionnelles, tout en les présentant formellement et systématiquement suivant un ordre convenu, fondé sur les parties du discours: noms, verbes, adjectifs, adverbes, prépositions, conjonctions, interjections, etc.; les éléments de formation (affixes et formants savants) clôturent chaque article, le cas échéant (voir les échantillons d'articles en annexe). Les catégories lexico-grammaticales gèrent donc la microstructure des articles. Chaque catégorie d'unité lexicale est précédée d'un code abréviatif apparaissant dans la marge, tandis que chaque changement de sens amène un nouveau sous-paragraphe qui se démarque par un alinéa et un numéro d'ordre. Pour chaque notion, les sous-paragraphes sont successivement numérotés de 1 à $n$ (par exemple, 383 Maladie possède 86 de ces alinéas - déployés sur 11 colonnes; 576 Rapidité en a 56; 666 Manœuvres en a 45; 713 Droit en a 63). La plupart des articles dépassent largement la dizaine d'alinéas.

Le mot-phare sert à regrouper les unités par famille morphologique et sémantique. Si l'on se reporte à l'article 33 Variation, on y croise les sous-entrées N. Variation (9 sens), 
V. Varier (4 sens), Adj. variable ( 4 sens), Adv. Variablement (1 sens) et les 5 affixes sémantiquement liés à ce concept : apo-, multi-, poly-, precilo-, poïkilo-. Dans le contexte de ce dictionnaire, le terme sens doit être interprété dans une acception particulière, car on n'y rencontre pas de définition élaborée de type phrastique. Le «sens» est toujours représenté par une seule autre unité lexicale, par un mot ou un terme complexe ou encore par une locution ou une expression figée. De fait, il s'agit bien davantage de synonymes que de paraphrases définitionnelles.

Un appareil diacritique facilite le décodage de chaque énoncé de sous-paragraphe. Le tiret note une division sémantique forte, tandis que le point-virgule indique des nuances de sens plus fines (voir 33 Variation: V.9 Varier; changer 193, fluctuer, muer; tourner à, virer à. - Devenir.). Il y a ici deux séries de verbes séparées par un tiret. La première renvoie aux synonymes de varier ayant la plus grande extension: changer, fluctuer, muer; tourner $\grave{a}$, virer $\grave{a}$; la deuxième série recouvre un autre changement sémantique, celui de «devenir». À l'intérieur de chaque séquence, les unités sont agencées en ordre alphabétique, sauf dans de rarissimes cas, comme celui des planètes où la disposition naturelle par rapport au soleil a prévalu sur les signes linguistiques. D'autres codes microstructurels complètent la physionomie d'un article: les caractères gras, qui mettent en évidence les mots les plus usuels, ou dont le sens est très général, ou qui se distinguent par une valeur stylistique notable, les renvois numérotés, qui encouragent le lecteur à poursuivre ses recherches dans un réseau notionnel en établissant une passerelle entre les différentes familles conceptuelles aux fins de faciliter les associations d'idées ou de favoriser la quête du mot le plus apte à rendre la pensée, les indicatifs de marques d'usage avec lesquels les consulteurs sont familiers - registres sociaux, temporels, géographiques (belgicisme, canad[ianisme], québécisme et régionalisme sont repérables dans Ia liste des abréviations; helvétisme en est absent), professionnels (les abréviations de 120 domaines spécialisés figurent dans la liste idoine), etc.

Respectant en cela la légendaire tradition de prudence laroussienne, la description des régionalismes ne va pas au-delà d'un petit nombre de mots sécuritaires et sur lesquels personne ne chicane (voir cependant 306.7 Inuits, forme du pluriel français tout à fait naturelle qui indique que l'emprunt est parfaitement intégré, et que, personnellement, je n'hésite pas à entériner). Les quelques québécismes généraux ou technolectaux suivants, relevés au hasard dans les nouvelles de ce jour, n'apparaissent pas dans le TL: bleuet (alors que myrtille et son synonyme régional (Normandie, Est, Provence) brimbelle ainsi que l'homonyme floral sont enregistrés), cégep et polyvalente (l'helvétisme gymnase y est tandis que le belgicisme athénée est absent), crème glacée (seul glace est cité), inhalothérapie, maganer, parcomètre (à côté de parcmètre qui est noté), rang, taponner. La fréquence d'usage de ces québécismes n'est pourtant plus à démontrer! De sorte que l'on peut conclure qu'en dépit d'une timide ouverture à la francophonie extra-hexagonale, la nomenclature entière du TL est d'abord et avant tout gallo-française.

\section{L'INDEX (PP. 631-1 146)}

L'index réunit 100000 «mots» : noms communs, noms propres (de lieux, de personnes, les marques déposées, etc.), affixes français et formants gréco-latins, tous rangés alphabétiquement, à raison de quatre colonnes par page. Ces mots permettent de retracer les différents paragraphes du dictionnaire où sont assemblées les unités lexicales qui réfèrent à la même idée ou à des idées apparentées. Sous chaque unité de l'index sont notés les sens, réduits à une version synonymique, et, s'il y a lieu, les rattachements à des terminologies thématiques ou à des syntagmes généraux ou spécialisés, à des locutions ou expressions, chaque information étant suivie d'un numéro séquentiel auquel elle est affiliée dans le thésaurus. Voici deux exemples d'entrée de l'index: 
variation 33

modification, changement $25.4 ; 193.1$;

522.3

variante $23.4 ; 62.3$;

76.5

ornementation musi-

cale $781.25 ; 781.32$

t. de chorégraphie

786.14

variations saisonnières

273.8

\author{
veau $397.7 ; 816.2$ \\ veau d'or 260.14 \\ adorer le veau d'or \\ $709.5 ; 829.11$ \\ tuer le veau gras \\ $855.23 ; 590.7$
}

Le recours à l'index est obligatoire pour qui désire circuler rapidement, systématiquement et efficacement dans le dictionnaire et ainsi accéder aux variantes linguistiques possibles pour exprimer une idée. La logique de consultation transite par l'index, qui est le véritable truchement dictionnairique de l'ouvrage, et cela en vertu du rigide et toujours commode classement alphabétique qu'on y retrouve. Paradoxalement, ce système possède à la fois des attributs positifs et des aspects négatifs. Le va-et-vient permanent entre les deux parties principales du thésaurus est sans doute ce qui dérange le plus le consulteur débutant. C'est là le défaut majeur, malheureusement impossible à contourner, et inhérent aux œuvres de ce genre, notamment en raison de ce «surmoi cartésien» (p. VII) dont parle le responsable du TL et qui, semble-t-il, caractérise le peuple français. Même s'ils sont d'un maniement facile, les répertoires à double pôle heurteront toujours l'esprit linéaire des êtres humains. C'est une faiblesse bien compréhensible que de toujours vouloir suivre un cheminement rectiligne dans une recherche qu'on souhaite mener à terme le plus rondement possible. Seule la pratique répétée de l'œuvre amène à découvrir l'exceptionnelle richesse qu'elle renferme et son indéniable utilité pour les langagiers ou pour les enseignants et les étudiants qui se donnent la peine d'en feuilleter les pages.

Perçu dans une perspective historique, le Thésaurus Larousse complète la triade dictionnairique de cette grande entreprise lexicographique qui s'est d'abord intéressée à l'encyclopédie - grand livre sur le savoir qui dresse un bilan des connaissances humaines à une époque donnée - , puis aux dictionnaires de langue — porteurs de mots dont ils dessinent le portrait linguistique multiforme - auxquels on associera les répertoires hybrides ou les dictionnaires encyclopédiques qui peignent à la fois les mots et les choses, enfin, à l'approche du XXI $\mathrm{XX}^{\mathrm{e}}$ siècle, au trésor conceptuel qui convoque simultanément les idées et les mots.

Larousse met à la disposition de ses fidèles et de ses nouveaux adeptes un répertoire d'une facture très particulière, unique en son genre pour la langue française. Théoriquement, le dictionnaire général monolingue fonctionne dans une seule direction; il cartographie prioritairement les mots selon leurs différentes configurations linguistiques, à l'exclusion d'autres aspects, du moins en principe. C'est un livre-outil sur le langage. Tandis que le thésaurus fait circuler les éléments consignés dans les deux sens : son caractère de bidirectionnalité favorise le passage du mot à la notion ou de l'idée à son ou à ses expressions linguistiques. Deux voies d'accès, le sommaire et l'index, servent de cordons ombilicaux au dictionnaire. L'un ne va pas sans les autres tant ils sont indissociables. On pourrait les comparer à l'un de ces rythmes ternaires si chers à Gustave Flaubert. Le TL est une somme de signes réunis sous le grand parapluie onomasiologique et dont les facettes signifiantes et signifiées sont en interaction constante.

L'utilité la plus immédiate du Thésaurus Larousse est bien évidemment axée sur l'aide à l'écrit. Quel mot correspond le mieux à telle idée qui demande à être davantage 
précisée? Quelle forme linguistique semble la plus adéquate dans tel environnement linguistique? Quelle association novatrice permet d'éviter les clichés, les collocations banales? Existe-t-il d'autres verbes que être, avoir ou faire en français? Etc. S'il ne dispense pas l'apprenant ou le rédacteur aguerri de la consultation du dictionnaire de langue classique, le TL ouvre ses pages et ses colonnes au lecteur curieux, patient et soucieux de la qualité de sa langue et de son style. La quête de la matière pour ce compte rendu m'a vite familiarisé avec ce nouvel outil lexicographique et elle m'en a révélé tout l'intérêt et toute la valeur. Tout au long de la rédaction, j'ai puisé allègrement et abondamment dans ce réservoir inépuisable d'idées et de mots. En toute justice, je dois dire que le présent compte rendu doit beaucoup à cette splendide et indispensable trouvaille dictionnairique qui, en maillant les mots et les idées, forme à la fois la chaîne et la trame de la langue française.

\section{ANNEXE}

Quelques échantillons d'articles du TL

\section{NORME}

I I Norme; anon, sandard. - Arehtype moddle, parangon 31. - Milies, juste milieu, moyenne (la moverme), normale (ha nomale), ordinaire ("ordinaire).

2 Critere, référeace, valeur; idell - Benu (le bean) 136 ; bien (le bien) 690 ; bod the bom), le bon usage - Deontologie.

3 Calibre, énlon - reon : forme, gabarit, pacron, purtem lanplich type - Balance,! regule [rean, ane]

4 Nome de Eabriation (nome AFNOR, nome Eanpaise ou NA); norme de producrivite.

5 Canonicite [tidat)] conformite, noma. lité, nommativite (didser], régularites; 16galite 713. - Crammaticalite [luna]

6 Validité.

7 Normalisition, rígularisation, smandardisation; unifeacion. - Systefmatisation [didact]. - Régulation.

8 Codification, reglementention; IGgalise tion. - Modelisation.

9 Calibrage, talonoage, formange [roopax]

v. 10 Normer; Gxer une norme - Codifier, reglementer; ligiftrer. - Definir. - Breveter.

11 Metre aux normes, normaliser, rtgulariser, standardiser; roguler, unifie. Adapter, ajuster, approprier, conformer; barmoniser; thire cadra avec, stgier sur.

12 Calibrer, tulonoer; formater [oromel] modeliser.

13 Se conformer a, se rigler sur; se plier a, se soumeture 2. - Rencere dans le rang revenir a la norme. - Faire comme tout le monde.

\section{CERCLE}

M I Cercle; boucle, circonference, disque, rond (un rond); cycle 185, orbe [ntt] Circularité, rocondité, sphéricité [didect].

2 Anneau, anneles, bague 866. - Caree [TECHN] cerceau, couronne. - Roue; rouelle, roadetle

3 Spheroïde (en sheroide)

4 stron : orbite; colure, Ecliptique, epicyde [anc]. - Equateur, tropique; mér. dien, parallile. - eecor : cercle anearecique, cencle arerique. - Cercle déférear ou déférent [anc].

5 Disque ; artole, aurtiole, cerne, nimbe Gloire, mandote $[\mathrm{ax}-\mu]$ - Rosace, rose [Bx-A]. - Astron : faux-soleil 277, balo, parasélène, parhélie; anneaux ou anses de Saturne [vx]

6 Circuit 858, circulation [vx], cincumanigation, périple; tour, toumée. - Révolution, rotation 215 ; circumduction [sc.]

7 Cercle infernal, cercie vicieurx - Cerde vertueux [econi].

8 Cyele ; cercie (cercle des saisons) 187 ; eternel sctour.

9 Sphère; boule, globe. - Balle, bille, alot - Ballonnet, baudnuche. - Cylindre, rouleau, nmbour.

10 Amphithéatre. - Cirque 270. - Rotonde 738 ; coupole, tambour.

il Baguage, cerclage. - Eocerclement

v. 12 Cerclex ; baguer, cylindrer. - Aureoler, cernes, couronner, nimber; ceindre, encercler, encoures.

13 Circuler; boucler, faire le tour de, tourner. toumer en rond. - La boucle ert boucles [oce cour.]- - Firre le cercle; ; entrex 
A4 14 Modale, standard, type. - Coutumier 685 ; usitt, usuel; d'usage, en usage.

15 Naturel, normal; moyen, ordinaire.

16 Conforme, valide; canonique, codique [didact]. - Correct; bon, exact, juste.

17 Normatif [didact]

18 Normé 246.

Aor. 19 Selon ou suivant la norme. - Legalement, légicimernent.

Aea.20 D'apres, selon, suivant; en conformite aver; conformément 3

All. 21 Nomo-, norm-, normo-, orth-, ortho, typo-

22 -nume, -type, -typie. dans la ronde.

ar it Circulaire; rond, rownd [itt]; spherique; orbitaire, orbial. - Circonftrentiel ; concentrique, excentrique. - Annulaire, oylindrique, ubulaire.

15 Annele, aréolaire, eirciné towe.l coutronné.

nov. 16 Circulairement. - En cercie, en rond; en circuit fermé, en circuit ouvert.

Alt 17 Cyclo-; Ov-, ovo-; circum-; péri-; gyro-.

18 -cycle.

\section{DEPASSEMENT}

N 1 Depassement; devancement, distanciation; debordement - Doublement Eloignement 200.

2 Avance, avancte, saillic 204 ; empittemenc - Echappé.

3 Limite 136; borne.

4 Depassement de soi, effort 530.

v. 5 Depasser; deborder; doubler, passer devant; diseancer 162, hisser derriere soi, hisser loin derritere soi; forlonger [Vul] - Outrepasser; franchir. - Dépasser (ou : doubler, tranchir, passer) le cap de; aller au-dela de.

6 Devancer; décrarnponner tan ou SPCATS], gratter [SPOATS]; tam : lacher, semer.

7 S'echapper, se détacher ; prendre la tête.
8 Depasser, empiéter, saillir.

9 Dépasser la mesure, dépasser les limites; aller trop loin, passer (ou : dépasser, franchir) les bornes 80, sorir des limites. 1. 10 Hors-limite. - Defbordant.

11 Depassé ; démodé, désuet 196, périmé. Aov. 12 Devant; en avant 163.

Prep 13 Aundeli de, en dehors de, hors de; pardela.

Coni 14 Apres que.

At $15 \mathrm{Ex}$-, exo-, hors-. 